\title{
An automatic analysis method of utterance and pause lengths and frequencies
}

\author{
YOSHIYUKI HORII \\ University of Colorado, Boulder, Colorado
}

\begin{abstract}
Automatic analysis of the lengths and frequencies of utterance and pauses is highly desirable in the study of large amounts of speech produced by pathologic speakers and of interactive speech such as that between mother and child, clinician and client, and teacher and student. This paper describes a microcomputer-based analysis method of utterance and pause lengths and frequencies in connected speech. The instrumentation consists of a rectifier-smoother circuit, an analog-to-digital convertor and an Apple microcomputer. The printouts list utterances and pauses (in milliseconds) in the order of occurrence, and means and standard deviations of the utterances and pauses. The program and the results of reliability and validity tests of this method are described.
\end{abstract}

The study of utterance and pause durations and their frequencies of occurrences in human speech provides important insights into the normal processes of speech production and perception as well as into pathologic conditions such as stuttering, dysarthria, aphasia, apraxia, laryngectomy, deafness, and other speech, language, and hearing impairments (Black, Tosi, Singh, \& Takefuta, 1966; Love \& Jeffress, 1971; Love, Starbuck, \& Christensen, 1972; Prosek \& Runyan, 1982; Reich, 1980; Ruder \& Jensen, 1972; Tanner, 1976). In addition, recent research on pauses in interactive speech between teacher and student, interviewer and interviewee, clinician and client, and mother and child has made the accurate and efficient measurements of utterance and pause lengths in dialogue imperative (Clemmer, 1980; Grosjean \& Lane, 1974, 1976; Kowal, O'Connell, \& Sabin, 1975; Rowe, 1974; Ruder, Jensen, \& Brandt, 1970; Siegman, 1978). However, traditional analog methods of utterance and pause length analysis, such as the use of oscillographs, spectrographs, and graphic level recorders, are expensive, time-consuming, laborious, and, in particular, not well suited for analysis of large numbers of data. Specialized hardware devices developed by some researchers are often costly and typically involve further treatment and/or verification of the results (Goldman-Eisler, 1968; O'Connell \& Kowal, 1981; Tosi, 1965). Current accessibility of microcomputers in many laboratories, clinics, and schools makes feasible an alternative microcomputer-based method of utterance and pause analysis. A microcomputer-based method, limited to the pause analysis only, was reported by Gooding, Gooding, and Swift (1982).

The author is affiliated with the Department of Communication Disorders and Speech Science, University of Colorado, Boulder, Colorado 80309.

\section{PROBLEM}

Initially, automatic analysis of utterance and pause appears to be simple. For example, pause may be defined as any segment of the smoothed speech signal that has an amplitude of $x$ milliV or less and a minimum duration of y millisec (Rochester, 1976). Utterances can be defined as all the nonpause segments. Applications of these definitions to speech signals having appropriate amplitude thresholds and minimum pause durations, however, would immediately reveal that pauses are often segmented into smaller pieces because of brief ambient or instrumental noise bursts. Raising the amplitude threshold in an at tempt to avoid such noises, on the other hand, systematically underestimates utterance durations and overestimates pause durations. Thus, a strategy to reject such transient noises during otherwise unitary pauses without raising the amplitude threshold is crucial and needs to be implemented in any automatic procedure.

Assuming that noise transients during pauses are, in fact, brief in comparison with real utterances, one could modify the basic definitions to include a noise rejection criterion such that utterance segments of less than, say, $100 \mathrm{msec}$ would be reclassified as a part of their preceding and following pauses, thus collapsing the measures. Together with the criteria of the amplitude threshold (minimum utterance and pause duration), the modified operational definitions appear to work reasonably well in identifying the utterance and pause segments in the connected speech. The purpose of this paper is to present a microcomputer-based automatic method of utterance and pause analysis that employs such an approach described above, and to provide some reliability and validity data for this method.

\section{METHOD}

The basic instrumentation for utterance and pause analysis is illustrated in Figure 1. The initial stage consists of signal condi- 


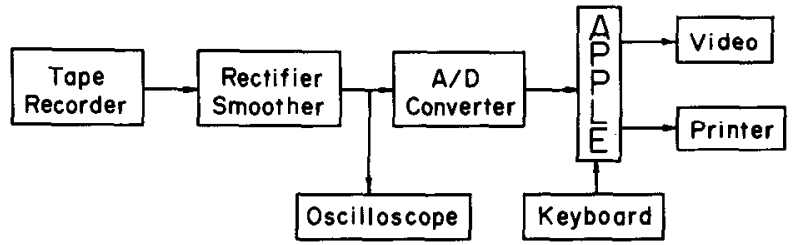

Figure 1. A block diagram illustrating the microcomputerbased analysis method of utterance and pause lengths and frequencies.

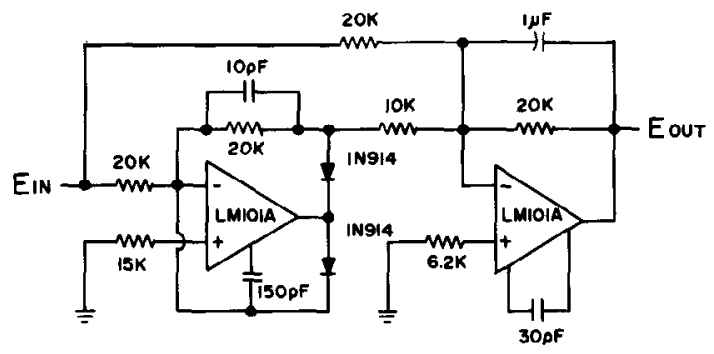

Figure 2. A circuit diagram of the rectifier-smoother for extracting speech amplitude envelope.

tioning by a rectifier-smoother. (A circuit diagram is shown in Figure 2.) The rectifier-smoother extracts amplitude envelopes of the speech signal by full-wave rectification and low-pass filtering. The extracted envelope signal is then analog-to-digital converted (TecMar AD211 12-bit convertor) at a sampling rate of 1,000 times/sec. A computer program written for an Apple II Plus microcomputer keeps track of occurrences and durations of the segments above and below the amplitude threshold specified by the experimenter. At the end of sampling, which is cuec by the experimenter's pressing the spacebar on the keyboard, the program scans through and, if necessary, collapses the segmental data using the durational criteria initially specified by the experimenter. The printout shows the utterances and pauses (in milliseconds) in the order of occurrence, and their means and standard deviations.

Reliability and validity tests were conducted using (1) square pulses and (2) speech signals. Square pulses of 1 and 10 pulses/ $\mathrm{sec}$ were generated by a function generator (Hewlett-Packard Model 3312A, monitored on a Tektronix 5441 storage oscilloscope). The pulse signals bypassed the rectifier-smoother circuit and were analog-to-digital converted for analysis. Computer results were obtained for 20 pulses each for the two pulse-rate conditions. For the speech analysis, tape-recorded voice samples of four adult males reading a 98-word passage ("The Rainbow Passage," by Fairbanks, 1960) were analyzed using the computer method and a graphic level recorder (Bruel and Kjaer Type 2305, with a paper speed of $100 \mathrm{~mm} / \mathrm{sec}$ and a writing speed of $400 \mathrm{~mm} /$ sec in RMS mode) for comparison. Durational measurements from the graphic level tracings were made by two independent observers, who used metric rulers to measure (to an accuracy of $0.5 \mathrm{~mm}$ ) distances and converted these values into milliseconds. Perceptual identification of pauses in the voice samples was made with unanimous agreement by three judges, who listened to the recorded samples as often as necessary to locate the pauses.

\section{RESULTS AND DISCUSSION}

The results of the computer analysis of the square pulses revealed that the maximum size of the difference between the computer data and oscilloscopic measure- ments for both pulse-rate conditions was $2 \mathrm{msec}$ and that the average difference was $0.3 \mathrm{msec}$. These magnitudes of difference were within an expected range when a sampling rate of 1,000 times/sec was employed. In terms of the percentage difference, the measured durations had the maximum difference of $0.4 \%$ for the 1-pulse/sec signal and $2 \%$ for the 10-pulses/sec signal. These results indicated that the software timer/counter of the program was appropriately precise.

The perceptual pause identification indicated that the three listeners unanimously agreed upon a total of 39 pauses in the readings by the four speakers. All of these perceived pauses were identified as pauses by the computer method as well. The average durations of these pauses and utterances, as measured by hand by the two observers from the graphic level tracings, were compared with the results obtained by the computer method and are presented in Table 1. (Table 1 also permits examination of the differences of durational measures by the two methods.) The average difference between the two methods was $20.2 \mathrm{msec}$, with a maximum difference of $66 \mathrm{msec}$. A Pearson product-moment correlation coefficient of $r=0.999$ was achieved between the two sets of data.

In order to examine the larger discrepancy between the computer method and hand measurements for the speech signals than for the square pulses, further experimentation was conducted with different writing speeds of the graphic level recorder and different amplitude thresholds. The results revealed that the large discrepancy was primarily attributable to the differences in

Table 1

Results of Utterance (U) and Pause (P) Analysis (in Milliseconds) of the Oral Readings of the Rainbow Passage by Four Adult

Speakers Using the Computer Method (Comp) and the Hand Measurements (Hand) From the Graphic-Level Tracings. The Absolute Differences (Diff) Between the Two Methods are Also Shown

\begin{tabular}{lrrr}
\hline & Comp & Hand & Diff \\
\hline U1 & Speaker 1 & \\
P1 & 4624 & 4610 & 14 \\
U2 & 544 & 540 & 4 \\
P2 & 3421 & 3405 & 16 \\
U3 & 611 & 635 & 24 \\
P3 & 449 & 450 & 1 \\
U4 & 201 & 175 & 26 \\
P4 & 3283 & 3270 & 13 \\
U5 & 372 & 375 & 3 \\
P5 & 2488 & 2475 & 13 \\
U6 & 618 & 625 & 7 \\
P6 & 1686 & 1675 & 11 \\
U7 & 291 & 290 & 1 \\
P7 & 2270 & 2260 & 10 \\
U8 & 415 & 400 & 15 \\
P8 & 594 & 635 & 41 \\
U9 & 373 & 365 & 8 \\
P9 & 1307 & 1295 & 12 \\
U10 & 536 & 525 & 11 \\
P10 & 2283 & 2290 & 7 \\
U11 & 443 & 435 & 8 \\
\hline
\end{tabular}


Table 1 (Continued)

\begin{tabular}{|c|c|c|c|}
\hline & Comp & Hand & Diff \\
\hline \multicolumn{4}{|c|}{ Speaker 2} \\
\hline U1 & 2543 & 2520 & 23 \\
\hline $\mathrm{P} 1$ & 594 & 605 & 11 \\
\hline $\mathrm{U} 2$ & 2282 & 2265 & 17 \\
\hline P2 & 1158 & 1170 & 12 \\
\hline U3 & 3508 & 3485 & 23 \\
\hline P3 & 1179 & 1195 & 16 \\
\hline U4 & 2427 & 2395 & 32 \\
\hline P4 & 903 & 915 & 12 \\
\hline U5 & 1429 & 1405 & 24 \\
\hline P5 & 621 & 635 & 14 \\
\hline U6 & 2644 & 2615 & 29 \\
\hline P6 & 1183 & 1185 & 2 \\
\hline U7 & 1943 & 1945 & 2 \\
\hline P7 & 212 & 185 & 27 \\
\hline U8 & 1298 & 1310 & 12 \\
\hline P8 & 1098 & 1080 & 18 \\
\hline U9 & 1814 & 1840 & 26 \\
\hline P9 & 1392 & 1385 & 7 \\
\hline U10 & 2421 & 2395 & 26 \\
\hline P10 & 761 & 770 & 9 \\
\hline U11 & 3561 & 3525 & 36 \\
\hline \multicolumn{4}{|c|}{ Speaker 3} \\
\hline U1 & 2911 & 2860 & 49 \\
\hline P1 & 547 & 615 & 41 \\
\hline $\mathrm{U} 2$ & 3173 & 3155 & 18 \\
\hline P2 & 804 & 820 & 16 \\
\hline U3 & 3899 & 3850 & 49 \\
\hline P3 & 822 & 850 & 28 \\
\hline U4 & 4495 & 4445 & 50 \\
\hline P4 & 534 & 550 & 16 \\
\hline U5 & 3277 & 3240 & 37 \\
\hline P5 & 747 & 760 & 13 \\
\hline U6 & 1822 & 1800 & 22 \\
\hline P6 & 525 & 535 & 10 \\
\hline U7 & 2761 & 2735 & 26 \\
\hline P7 & 638 & 640 & 2 \\
\hline U8 & 617 & 650 & 33 \\
\hline P8 & 289 & 285 & 4 \\
\hline U9 & 1808 & 1775 & 33 \\
\hline P9 & 827 & 845 & 18 \\
\hline U10 & 2677 & 2655 & 22 \\
\hline P10 & 687 & 695 & 8 \\
\hline U11 & 4952 & 4910 & 42 \\
\hline \multicolumn{4}{|c|}{ Speaker 4} \\
\hline U1 & 2518 & 2505 & 13 \\
\hline P1 & 553 & 565 & 12 \\
\hline U2 & 2513 & 2475 & 62 \\
\hline P2 & 555 & 585 & 30 \\
\hline U3 & 1727 & 1720 & 7 \\
\hline P3 & 217 & 230 & 13 \\
\hline U4 & 2274 & 2230 & 44 \\
\hline P4 & 610 & 615 & 5 \\
\hline U5 & 4103 & 4085 & 18 \\
\hline P5 & 389 & 395 & 6 \\
\hline U6 & 2555 & 2535 & 20 \\
\hline P6 & 527 & 545 & 18 \\
\hline U7 & 3640 & 3605 & 35 \\
\hline P7 & 462 & 475 & 13 \\
\hline U8 & 2097 & 2105 & 8 \\
\hline P8 & 621 & 605 & 16 \\
\hline U9 & 2166 & 2100 & 66 \\
\hline $\mathrm{P9}$ & 454 & 515 & 61 \\
\hline U10 & 4005 & 3975 & 30 \\
\hline
\end{tabular}

the smoothing characteristics of our hardware device and those of the level recorder, and secondarily to the less favorable signal-to-noise amplitude ratios of the speech than of the square pulses. To a lesser degree, the precision of the hand measurement, that is, $0.5 \mathrm{~mm}$, which corresponds to 5-msec accuracy, and the precision of the paper speed of the graphic level recorder contributed to the discrepancy between the two methods for the speech-signal analysis.

This automatic method of utterance and pause analysis requires that the experimenter make initial specifications of the three variables: (1) the amplitude threshold, (2) the minimum utterance duration, and (3) the minimum pause duration. Among these, the effects of amplitude threshold on durational measures are appreciable and need to be determined with caution. Most professional recordings have approximately a $40-\mathrm{dB}$ speech-to-noise ratio, whereas typical recordings made at home or in the office, classroom, etc., and not in a sound-attenuated booth or studio often have a 20- to 30- $\mathrm{dB}$ speech-to-noise ratio. It is apparent that recordings with poor speech-to-noise ratios, regardless of the analysis method, result in the underestimation of utterance lengths and overestimation of pause durations. It is crucial, therefore, to strive for the best possible recording conditions. Occasionally, it is possible to improve the speech-to-noise ratio of poor recordings by filtering out noise if its spectrum lies outside the range of primary speech spectra.

In the present experiment, the minimum utterance length of $100 \mathrm{msec}$ and the minimum pause duration of $200 \mathrm{msec}$ were specified. For some applications, much smaller minimum utterance or pause durations might be desired. For example, silent gaps in stutterers' speech are often $100 \mathrm{msec}$ or less (Love \& Jeffress, 1971; Prosek \& Runyan, 1982; Rieber, Breskin, \& Jaffe, 1972). Minimum utterance durations of poor esophageal speakers, on the other hand, can be less than $100 \mathrm{msec}$. The experimenter-specification feature of the variables in this automatic method makes it applicable to both types of speech analysis.

Note that the present method analyzes speech in terms of presence and absence of physical gaps in the signal. Thus, the pause in this context refers to the socalled unfilled pauses. Hesitation pauses with overt vocalizations such as "uh ..." or "humm ...", therefore, are treated as utterances if they are long enough, that is, if they exceed the specified minimum utterance duration. Perceptual experiments currently being conducted also indicate that the durations of the physical gaps are not necessarily related to perceptually identified pauses. In other words, both the linguistic content and the articulatory characteristics of the speaker appear to affect the perception of unfilled pauses, and a $100-\mathrm{msec}$ gap may be perceived as pause in one context and as nonpause in another context. A systematic investigation of silent gaps in speech and perception of pauses is currently in progress in our laboratory. 
Summary of the Program Functions

The program (1) samples rectified and smoothed speech -signals at 1,000-times/sec, (2) stores segment durations above and below a specified amplitude threshold in the order of occurrence, (3) collapses the segments, if necessary, according to the specified minimum durations of utterance and pause, (4) prints the utterance and pause durations (in milliseconds) in the order of occurrence, and (5) prints means and standard deviations of utterance and pause durations. The main program was written in BASIC; the analog-to-digital conversion routines were written in machine language.

\section{Memory Requirement}

A minimum of $16 \mathrm{~KB}$ of memory is required.

\section{Program Availability}

Program listing is available free. Send a self-addressed stamped envelope to: Yoshiyuki Horii, Campus Box 409, Department of Communication Disorders and Speech Science, University of Colorado, Boulder, Colorado 80309.

\section{REFERENCES}

Black, J., Tosi, O., Singh, S., \& Takefuta, T. A study of pauses in oral reading of one's native language and english. Language and Speech, 1966, 9, 237-241.

Clemmer, E. J. Psycholinguistic aspects of pauses and temporal patterns in schizophrenic speech. Journal of Psycholinguistic Research, 1980, 9, 161-185.

Fairbanks, G. Voice and articulation drillbook. New York: Harper, 1960.

Goldman-Eisler, F. Psycholinguistics: Experiments in spontaneous speech. London: Academic Press, 1968.

Gooding, S. T., Gooding, C. T., \& SwiFT, J. N. A microcomputer-based pause analysis apparatus. Behavior Research Methods \& Instrumentation, 1982, 14, 121-123.

Grosjenn, F., \& Lane, H. Effects of two temporal variables on the listener's perception of reading rate. Journal of Experimental Psychology, 1974, 102, 893-896.

Grosjean, F., \& Lane, H. How the listener integrates the com- ponents of speaking rate. Journal of Experimental Psychology, $1976,2,538-543$.

Kowal, S., O'Connell, D. C., \& Sabin, E. J. Development of temporal patterning and vocal hesitations in spontaneous narratives. Journal of Psycholinguistic Research, 1975, 4, 195-206.

LOVE, L. R., \& JeFFress, L. A. Identification of brief pauses in fluent speech of stutterers and nonstutterers. Journal of Speech and Hearing Research, 1971, 14, 229-240.

Love, L. R., Starbuck, H. B., \& Christensen, J. M. Pause frequency in fluent and nonfluent speech. Journal of Acoustical Society of America, 1972, 51, 122-123.

O'Connell, D. C., \& Kowal, S. Pausology. In W. Sedlow \& S. Sedlow (Eds.), Computer uses in the study of languages (Vol. 3): Cognitive approaches. The Hague: Mouton, 1981.

Prosek, R. A., \& Runyan, C. M. Temporal characteristics related to the discrimination of stutterer's and nonstutterer's speech samples. Journal of Speech and Hearing Research, 1982, 25, 29-33.

REICH, S. S. Significance of pause for speech perception. Journal of Psycholinguistic Research, 1980, 9, 279-289.

Rieber, R. W., Bresinin, S., \& Jaffe, J. Pause time and phonation time in stuttering and cluttering. Journal of Psycholinguistic Research, 1972, 1, 149-154.

Rochester, S. R. Defining the silent pause in speech. Journal of Ontario Speech and Hearing Association, 1976, 8, 1-4.

Rowe, M. B. Pausing phenomena: Influence on the quality of instruction. Journal of Psycholinguistic Research, 1974, 3, 203-224.

Ruder, K. F., \& Jensen, P. J. Fluent and hesitation pauses as a function of syntactic complexity. Journal of Speech and Hearing Research, 1972, 15, 49-60.

Ruder, K. F., Jensen, P. J., \& Brandt, J. F. An apparatus and procedure for the perceptual study of speech pauses. Journal of Experimental Analysis of Behavior, 1970, 14, 287-289.

Siegmen, A. W. The meaning of silent pauses in the initial interview. Journal of Nervous and Mental Disorders, 1978, 166, 642.654.

TANNER, D. C. Spectrographic pausimetric and intelligibility measures in Parkinson's disease. Unpublished doctoral dissertation. Michigan State University, 1976.

Tosi, 0 . A method for acoustic segmentation of continuous sound into pauses and signals and measurement of segment durations. Unpublished doctoral dissertation, Ohio State University, 1965.

(Manuscript received February 22, 1983; revision accepted for publication May 6, 1983.) 